\title{
Extra-Articular Stabilization of the Canine Cranial Cruciate Ligament Injury Using Arthrex Corkscrew and FASTak Anchors
}

\author{
Erin N. Kishi ${ }^{1}$, Don Hulse ${ }^{2}$, Matthew Raske ${ }^{1}$, W. Brian Saunders ${ }^{2}$, Brian S. Beale ${ }^{3}$ \\ ${ }^{1}$ Capital Area Veterinary Specialists, Austin, USA \\ ${ }^{2}$ Department of Veterinary Small Animal Clinical Sciences, College of Veterinary Medicine, \\ Texas A\&M University, College Station, USA \\ ${ }^{3}$ Gulf Coast Veterinary Specialists, Houston, USA \\ Email: dhulse@cvm.tamu.edu
}

Received April 16, 2013; revised May 16, 2013; accepted June 9, 2013

Copyright (C) 2013 Erin N. Kishi et al. This is an open access article distributed under the Creative Commons Attribution License, which permits unrestricted use, distribution, and reproduction in any medium, provided the original work is properly cited.

\begin{abstract}
The objective of this study was to evaluate the long-term outcome in dogs with cranial cruciate ligament injury that were repaired with an Arthrex Corkscrew or FASTak bone anchor and Fiberwire placed at near isometric points of the stifle. A retrospective study (2006-2010) was conducted by reviewing clinical records of dogs treated for cranial cruciate ligament injury with an extra-articular repair method using anchors preloaded with Fiberwire. A questionnaire was given to owners for evaluation of their dog's performance prior to surgery and at time of follow-up with a minimum of 12 months post-operative. Owner assessment was rated using a visual analogue scale. Completed questionnaires from 34 owners were received. The mean time to follow up was 27.5 months. Owner assessment of their dog prior to surgery versus at the time of follow up were considered significant $(\mathrm{P}<0.001)$ in regards to quality of life, willingness to play voluntarily, activity level, stiffness at the beginning and end of the day, lameness in the surgical limb, and pain while walking on the surgical limb. These results indicate that extra-articular stabilization with Arthrex Corkscrew and FASTak anchors placed at near isometric sites are adequate repair methods for cranial cruciate ligament rupture.
\end{abstract}

Keywords: Corkscrew; FASTak; Cranial Cruciate Ligament Rupture; Extracapsular Repair; Questionnaire

\section{Introduction}

Complete or partial rupture of the cranial cruciate ligament (CCL) is a common injury of the canine stifle [1-3]. It is thought that mechanical loading leading to degenerative changes can be a factor in rupture under normal physiologic conditions [4], but likely it is a multi-factorial etiology. Cranial cruciate ligament injury causes cranial translation of the tibia relative to the femur resulting in hind limb lameness and often leads to osteoarthritis [5]. It has been demonstrated that dogs with CCL deficient stifles cannot prevent cranial translation of the tibia either by altering hind limb gait or muscle forces across the stifle [6]. As such, conservative treatment of CCL injury is generally unsuccessful. The majority of surgeons would agree that surgical stabilization is the preferred method of treatment [7]. Numerous surgical techniques have been developed including placement of intra-articular grafts, insertion of suture material and/or advancement of periarticular structures outside the joint (extra-articular), and tibial osteotomies that alter joint mechanics [8-10]. Although hind limb function and lameness can be improved with surgical intervention, to date, no one technique has proven to be superior in the clinical setting $[7,11]$. Procedures that require placement of an extra-articular suture are technically less demanding and remain popular with veterinary surgeons and veterinary practitioners alike. In vitro testing has demonstrated that techniques in which the stabilizing suture is secured to the bone rather than circumfabellar provide superior load to failure, stiffness and load to yield [12]. The purpose of this study is to report the long-term outcome of cranial cruciate ligament injuries stabilized with Arthrex FASTak ${ }^{1}$ and Corkscrew ${ }^{2}$ bone anchors placed at near isometric sites of the stifle [13]. Our hypothesis was that these an-

\footnotetext{
${ }^{1}$ Arthrex Inc., Naples, Florida, USA.

${ }^{2}$ Arthrex Inc., Naples, Florida, USA.
} 
chors placed near isometric sites would result in appropriate long-term functional outcome when applied to the clinical patient.

\section{Material and Methods}

Medical records from 2006-2010 were reviewed to identify dogs that had a cranial cruciate ligament rupture repaired with Arthrex FASTak or Arthrex Corkscrew anchors. For inclusion in the study, the following criteria were met for each case: lameness resulting from a partial or complete CCL rupture, treatment with a FASTak or Corkscrew anchor positioned at reported near isometric sites [13], completion of an owner telephone interview based on questions validated with force plate analysis [14], and a minimum of 12 months between surgery and telephone interview. Dogs that had surgery on both stifle joints during separate surgical dates were included as two separate patient interventions.

Signalment (sex, age, breed, body weight), partial or complete cranial cruciate ligament tear, meniscal damage, presence or absence of meniscal release, concurrent orthopedic diseases, type of bone anchor used for extraarticular stabilization and time to follow-up evaluation were recorded. Stifles were evaluated arthroscopically for documentation of intra-articular pathology and then stabilized using the surgical technique described by Hulse, et al. [15]. The $5.0 \mathrm{~mm}$ Corkscrew anchor was preloaded with \#5 Fiberwire ${ }^{3}$ and the $2.8 \mathrm{~mm}$ FASTak anchor was preloaded with \#2 Fiberwire. Post-operative radiographs were taken immediately after surgery to ensure anchor placement was in the correct location.

\subsection{Pre- and Post-Operative Care}

All patients were administered acepromazine ${ }^{4}(0.025 \mathrm{mg} / \mathrm{kg}$, intramuscularly), hydromorphone ${ }^{5}(0.1 \mathrm{mg} / \mathrm{kg}$, intramuscularly) and glycopyrrolate ${ }^{6}(0.011 \mathrm{mg} / \mathrm{kg}$, intramuscularly) as pre-medication. Induction of general anesthesia was initiated with propofol ${ }^{7}(5 \mathrm{mg} / \mathrm{kg}$, intravenously) to effect. Under general anesthesia, an epidural with preservative-free morphine ${ }^{8}(0.1 \mathrm{mg} / \mathrm{kg})$ was given as an adjunct analgesia. Cefazolin ${ }^{9}(22 \mathrm{mg} / \mathrm{kg}$, intravenously) was given as a perioperative antibiotic. Immediately postoperative, an intra-articular morphine ${ }^{10}(0.5 \mathrm{mg} / \mathrm{kg})$ block and a bupivacaine ${ }^{11}(5 \mathrm{mg})$ block around the incision were performed for local analgesia. Carprofen ${ }^{12}(4.4 \mathrm{mg} / \mathrm{kg}$ subcutaneously) was also given if the patient was not currently taking another non-steroidal anti-inflammatory.

\footnotetext{
${ }^{3}$ Arthrex Inc., Naples, Florida, USA.

${ }^{4}$ Boehringer Ingelheim Vetmedica Inc., St. Joseph, Missouri, USA.

${ }^{5}$ Baxter Healthcare, Deerfield, Illinois, USA.

${ }^{6}$ Baxter Healthcare, Deerfield, Illinois, USA.

${ }^{7}$ Hospira Inc., Lake Forest, Illinois, USA.

${ }^{8}$ Duramorph, Baxter Healthcare, Deerfield, Illinois, 60015, USA.

${ }^{9}$ West-Ward Injectables Inc., Eatontown, New Jersey, USA.
}

The patients remained in the hospital overnight and morphine $^{10}(0.5 \mathrm{mg} / \mathrm{kg}$, intramuscularly every 4 hours $)$ was given as needed for analgesia. Tramadol ${ }^{13}$ (3 - 5 $\mathrm{mg} / \mathrm{kg}$ per os QID) and a non-steroidal anti-inflammatory [carprofen (2.2 $\mathrm{mg} / \mathrm{kg}$ per os BID) or deracoxib ${ }^{14}(2$ $\mathrm{mg} / \mathrm{kg}$ per os daily)] were prescribed for 7 days for continued analgesia at home. Cephalexin ${ }^{15}(22 \mathrm{mg} / \mathrm{kg}$ per os BID) was given to continue antibiotics for 7 days. Postoperative care was standardized for each case and included specific exercise restriction and rehabilitation exercises for 7 weeks following surgery. Briefly, owners were advised to control activity when outside with a leash. When home and inside with the owner, supervised activity (no running, playing with other pets, jumping on and off furniture) was required. All cases underwent specific rehabilitation activity including aquatic therapy, weight bearing exercises, and balance activity.

A modified long-term follow-up questionnaire [14] was completed during a telephone interview with owners (Appendix A). Owners were queried regarding their dog's function following CCL injury and prior to surgery versus post-operative (current) function. If the patient was deceased at time of the interview, the time of follow-up was calculated from surgery to time of death (in months). A subjective scale of 1 to 6 was established $(1=$ worse, 6 = best) and used to answer each question prior to surgery and after treatment. Records were reviewed and owners questioned relative to the occurrence of postoperative complications.

\subsection{Statistical Analysis}

Statistical analysis was used to compare owner responses (number values) prior to surgery to owner responses (number values) at the time of follow-up. This was performed by use of the Wilcoxon signed-rank test. Values of $\mathrm{P} \leq 0.05$ were considered significant.

\section{Results}

Fifty-six dogs underwent surgery repair with a Corkscrew or FASTak anchor for a ruptured CCL during the 4.5-year period. Of the 56 cases, 34 dogs met the inclusion criteria and are included in the study. Twenty-two dogs did not satisfy inclusion criteria. Eighteen of the 22 dogs were lost to follow-up as their owners were unable to be contacted. Three of the 22 dogs had post-operative diseases that occurred within 12 months of surgery: One was euthanized 1 month post-operatively due to multiple

\footnotetext{
${ }^{10}$ Baxter Healthcare, Deerfield, Illinois, USA.

${ }^{11}$ Hospira Inc., Lake Forest, Illinois, USA.

${ }^{12}$ Rimadyl, Pfizer Animal Health, New York, New York, USA.

${ }^{13}$ Apotex Inc, Toronto, Ontario, Canada.

${ }^{14}$ Deramaxx, Novartis Animal Health, Greensboro, North Carolina, USA.

${ }^{15}$ Novopharm, Scarborough, Ontario, Canada.
} 
orthopedic problems, one dog died of throat cancer 5 months post-operative, and one dog died 2 months postoperatively of an undetermined cause. A single owner did not remember that her dog had stifle surgery.

Of the 34 dogs that met the inclusion criteria, there were 25 spayed females and 9 neutered males with a mean age of 7.7 years (range, 3 to 14 years). Several breeds were represented including Beagle (5), West Highland Terrier (5), Bichon Frise (2), Cocker Spaniel (2), Cairn Terrier (2), Pug (2), and Corgi, Border Collie, Chihuahua, Papillon, American Pitbull Terrier, Blue Heeler, American Spitz, Miniature Schnauzer and Maltese (1 each). There were also seven mix breed dogs included in the study population. The mean weight was $13.8 \mathrm{~kg}$ (range, 4.5 to $31.4 \mathrm{~kg})$.

Of the 34 cruciate ligament ruptures, 18 affected right hind limbs and 16 affected the left hind limbs. A complete cranial cruciate tear occurred in 24 of 34 (71\%) cases and a partial tear occurred in 9 of 34 (26\%). One dog had an avulsion of the ligament insertion. The caudal cruciate ligament was normal in all cases that were noted. A normal medial meniscus was seen in $25 \mathrm{dogs}$ and of these, 15 had a meniscal release. Nine dogs had a torn meniscus and therefore a partial meniscectomy was performed. The lateral meniscus was normal in all cases. Concurrent medial patella luxation was present in two dogs and one dog had a tear of the popliteal tendon.

Seven dogs underwent stabilization with the FASTak anchor preloaded with \#2 Fiberwire and 27 dogs underwent stabilization with the Corkscrew anchor preloaded with \#5 Fiberwire. Of the 34 dogs, one had two Corkscrew anchors placed, one had two \#5 Fiberwires placed with one Corkscrew anchor and one had two \#2 Fiberwires placed with one FASTak anchor. Two of the 34 dogs had concurrent medial patella luxations repaired: one had a wedge recession and lateral imbrication and the other dog had a block recession, tibial tuberosity transposition, medial release of retinaculum and lateral imbrication.

The mean length to follow-up was 27.5 months (range, 12 to 66 months). At the time of follow-up, four of the 34 dogs had died of unrelated causes or advanced age. Thirty-four owners were available and answered questions about their dog's status prior to surgery and after surgery. Results are reported in Table 1. Owner assessment of function prior to and after surgery were considered significant with regards to improvement in quality of life $(\mathrm{P}<0.001)$, willingness to play voluntarily $(\mathrm{P}<$ $0.001)$, activity level $(\mathrm{P}<0.001)$, stiffness at the beginning of the day $(\mathrm{P}<0.001)$, stiffness at the end of the day $(\mathrm{P}<0.001)$, lameness in the surgical limb $(\mathrm{P}<0.001)$, and pain while walking on the surgical limb $(\mathrm{P}<0.001)$.

Three of 34 (8.8\%) dogs had complications post-operatively: one complication was minor and two complications were major. The minor complication reported was an incisional dehiscence. Two dogs had major complications that necessitated reoperation: one dog had a bucket handle tear of the medial meniscus 9 months post-operative and the other dog had a reoperation to remove a torn \#5 Fiberwire (with a Corkscrew anchor) 3 months postoperative. An additional dog had a hemilaminectomy 3 weeks post-operative for intervertebral disc disease.

\section{Discussion}

In the present study, the Corkscrew and FASTak anchor systems appear to be an acceptable extra-articular stabilization procedure for stifles with cranial cruciate ligament disease in dogs with a mean weight of $13.8 \mathrm{~kg}$. Owner assessment of their dog's quality of life, activity level and limb function significantly improved after surgical stabilization.

The ideal extra-articular stabilization technique eliminates excessive cranial-caudal drawer motion, allows full stifle function and provides long-term stifle stability.

Table 1. Table summarizing the results of the owner questionnaire.

\begin{tabular}{|c|c|c|c|}
\hline Variable & Assessment prior to surgery & Assessment at time of follow up & Difference between groups \\
\hline Quality of life & $\begin{array}{l}\text { Mean: } 2.7 \\
\text { Median: } 2.5\end{array}$ & $\begin{array}{l}\text { Mean: } 5.6 \\
\text { Median: } 6\end{array}$ & $\begin{array}{l}\text { Statistically significant } \\
\mathrm{P}<0.001\end{array}$ \\
\hline Willingness to play voluntarily & $\begin{array}{l}\text { Mean: } 2.9 \\
\text { Median: } 3\end{array}$ & $\begin{array}{l}\text { Mean: } 5.5 \\
\text { Median: } 6\end{array}$ & $\begin{array}{l}\text { Statistically significant } \\
\qquad \mathrm{P}<0.001\end{array}$ \\
\hline Activity level during the day & $\begin{array}{l}\text { Mean: } 2.5 \\
\text { Median: } 2\end{array}$ & $\begin{array}{l}\text { Mean: } 4.9 \\
\text { Median: } 5\end{array}$ & $\begin{array}{l}\text { Statistically significant } \\
\qquad \mathrm{P}<0.001\end{array}$ \\
\hline Stiffness of affected limb at end of day & $\begin{array}{l}\text { Mean: } 3.1 \\
\text { Median: } 3\end{array}$ & $\begin{array}{l}\text { Mean: } 5.3 \\
\text { Median: } 6\end{array}$ & $\begin{array}{l}\text { Statistically significant } \\
\qquad \mathrm{P}<0.001\end{array}$ \\
\hline Lameness when walking on affected limb & $\begin{array}{l}\text { Mean: } 1.9 \\
\text { Median: } 2\end{array}$ & $\begin{array}{l}\text { Mean: } 5.5 \\
\text { Median: } 6\end{array}$ & $\begin{array}{l}\text { Statistically significant } \\
\qquad \mathrm{P}<0.001\end{array}$ \\
\hline Pain level when suddenly turning while walking & $\begin{array}{l}\text { Mean: } 2.6 \\
\text { Median: } 2\end{array}$ & $\begin{array}{l}\text { Mean: } 5.7 \\
\text { Median: } 6\end{array}$ & $\begin{array}{l}\text { Statistically significant } \\
\qquad \mathrm{P}<0.001\end{array}$ \\
\hline
\end{tabular}


Achievement of these points is dependent on factors that include the suture material, attachment sites of the suture at the femur and tibia, and the method used to secure the suture to the femur and tibia. Materials used for extraarticular sutures include monofilament nylon leader line or braided orthopedic suture. Both bone anchors in this study were preloaded with Fiberwire suture, a multistranded long chain ultra-high molecular weight polyethylene core with a braided jacket of polyester. Traditional suture placement is adjacent to the lateral fabella and near the insertion of the patella at the tibial crest. These locations can affect the isometry of the joint and therefore long-term cranial-caudal laxity of the stifle [13]. This has led to development of points of attachment that are more isometric than the traditional fabello-tibial suture sites. Near isometry was found to be two points that maintained a nearly fixed suture tension during full stifle range of motion and with increasing loads [13]. These sites were located at the caudolateral femoral condyle at the level of the distal pole of the lateral fabella (F2) paired with a site located at the bony protuberance $2 \mathrm{~mm}$ caudal to the sulcus of the long digital tendon (T3). Increasing tension to eliminate cranial drawer did not affect suture isometry at preloads tested [13].

Meniscal release was performed in 15 of 25 dogs that had a normal meniscus at the time of surgery. Release was performed as a surgeon preference based on age and the normal activity level the dog. Only one of the 10 dogs that did not have a meniscal release returned 9 months later with a torn medial meniscus. In a previous study [16], meniscal release did not significantly reduce the rate of subsequent meniscal tears in dogs where a tibial plateau leveling osteotomy was performed. The same results may not occur in dogs with an extra-articular repair and may have prevented more dogs from having postliminary meniscal tears in the current study. Further study is warranted to evaluate the outcome of meniscal release in dogs with a ruptured CCL where an extra-articular repair is performed.

Within this study, dogs that weighed less than $8.5 \mathrm{~kg}$ at the time of surgery were repaired with a FASTak anchor and \#2 Fiberwire and dogs that weighed greater than $8.5 \mathrm{~kg}$ at the time of surgery had a Corkscrew anchor and \#5 Fiberwire placed. The decision between these two anchor systems was made by the surgeon at the time of surgery, where the size of the femoral condyle F2 point was assessed. If the F2 point was too small for a $5 \mathrm{~mm}$ Corkscrew anchor, a $2.8 \mathrm{~mm}$ FASTak anchor was used. The selection of the size of the anchor used is a surgeon preference and therefore the $8.5 \mathrm{~kg}$ noted in this study is an arbitrary weight demarcation. It is possible that FASTak anchors could be used in dogs weighing greater than $8.5 \mathrm{~kg}$, but was beyond the scope of this study.

Complications with Arthrex FASTak anchors reported in human literature include suture breakage at the knot, rupture at eyelet and anchor pullout. Rupture at the eyelet of the $2.4 \mathrm{~mm}$ anchors occurred $61.5 \%$, while only $22.2 \%$ using the $2.8 \mathrm{~mm}$ anchors. This is thought to be due to the sharp inner edge of the smaller $2.4 \mathrm{~mm}$ anchor eyelets [17]. The bigger the eyelet diameter, the smoother the edges are. In addition, the breakage of suture material appears to depend on the orientation of the anchor eyelet relative to the loading direction of the suture thread [18, 19]. The newer suture anchors have shown to have markedly increased load to failure strengths to prevent anchor pullout as well [20]. But failure of the anchor may occur if not inserted properly. Although suture breakage did occur in one dog in this study, it did not occur as often as reported above [17]. It is possible that with a larger sample size, a larger incidence of suture breakage may have occurred.

In the present study, several limitations need to be considered when interpreting the findings. The retrospective nature and reliance on the owner recollection of preand post-operative lameness, pain and stiffness 5 years later may limit the conclusions that can be made from the results. In addition, the present study evaluated long-term outcomes using an owner assessment questionnaire. Although owner's perception of soundness may be variable, their ability to assess functionality can be reliable [14]. In the Hudson (2004) study, chronic lameness was quantified by force plate analysis and used as the criterion-referenced standard to ensure validity and reliability. In addition, dogs were available for test-retest assessment to further warrant reliability. By measuring multiple forces (total peak vertical, total vertical impulse and total peak propulsion), various aspects of lameness were recorded objectively. On the basis of statistical analysis of this data with repeatable questions, the questionnaire was found to be valid [14]. Kinetic gait analysis would have resulted in more objective data, but was not available at our hospital.

In conclusion, this retrospective study provides evidence that the Corkscrew and FASTak suture anchors are considered an effective and adequate extra-articular stabilization method in small to medium-sized dogs.

\section{REFERENCES}

[1] S. P. Arnoczky, "Pathomechanics of Ligament and Meniscal Injuries," In: Bojrab, Ed., Disease Mechanisms in Small Animal Surgery, 2nd Edition, Lea \& Febiger, Philadelphia, 1993, pp. 764-770.

[2] S. P. Arnoczky and J. L. Marshall, "The Cruciate Ligaments of the Canine Stifle: An Anatomical and Functional Analysis," American Journal of Veterinary Research, Vol. 38, No. 11, 1977, pp. 1807-1814.

[3] P. B. Vasseur, "Stifle Joint," In: D. Slatter, Ed., Textbook of Small Animal Surgery, 2nd Edition, Saunders, Phila- 
delphia, 1993, pp. 1817-1865.

[4] K. Hayashi, J. D. Frank, C. Dubinsky, et al., "Histologic Changes in Ruptured Canine Cranial Cruciate Ligament," Veterinary Surgery, Vol. 32, No. 3, 2003, pp. 269-277. doi:10.1053/jvet.2003.50023

[5] P. B. Vasseur, "Clinical Results Following Conservative Management for Rupture of the Cranial Cruciate Ligament in Dogs," Veterinary Surgery, Vol. 13, No. 4, 1984, pp. 243-246. doi:10.1111/j.1532-950X.1984.tb00801.x

[6] D. L. Korvick, G. J. Pijanowski and D. J. Schaeffer, "Three Dimensional Kinematics of the Intact and Cranial Cruciate Deficient Stifle of Dogs," Journal of Biochemanics, Vol. 27, No. 1, 1994; pp. 77-87.

[7] A. D. Elkins, K. Pechman and M. Herron, "A Retrospective Study Evaluating the Degree of Degenerative Joint Disease in the Stifle Joint of Dogs Following Surgical Repair of Anterior Cruciate Ligament Rupture," Journal of the American Animal Hospital Association, Vol. 27, No. 5, 1991, pp. 533-540.

[8] P. C. Gambardella, L. J. Wallace and F. Cassidy, "Lateral Suture Technique for Management of Anterior Cruciate Ligament Rupture in Dogs: A Retrospective Study," Journal of the American Animal Hospital Association, Vol. 17, No. 1, 1981, pp. 33-38.

[9] D. A. Hulse, "Cruciate Ligament Rupture," In: T. Fossum, Ed., Small Animal Surgery, 2nd Edition, Mosby, St. Louis, 2002, pp. 1110-1143.

[10] B. Slocum and T. Devine, "Cranial Tibial Wedge Osteotomy: A Technique for Eliminating Tibial Thrust in Cranial Cruciate Ligament Repair," Journal of the American Veterinary Medical Association, Vol. 184, No. 5, 1984, pp. 564-568.

[11] S. C. Roe, "Limitations of Cranial Cruciate Ligament Repairs: Prosthetic Ligament Substitutions," Proceedings of the 24th Annual American College of Veterinary Surgeons Veterinary Symposium, San Francisco, 3-6 November 1996.

[12] C. J. Choate, A. Pozzi, D. D. Lewis, et al., "Mechanical Comparison of Lateral Circumfabellar Suture, Tightrope CCL, and SwiveLock Bone Anchor for Extracapsular Stabilization of the Cranial Cruciate Ligament-deficient Sti-

\section{Appendix A: Owner Questionnaire}

Scale 1-6. 1 being the worst (low) and 6 being the best (high)

AFTER injury, but PRIOR to surgery

1) How was quality of life?

2) What was willingness to play voluntarily?

3) How was activity during the day?

4) Stiffness at the beginning of the day?

5) Stiffness at the end of the day?

6) Indication of lameness when walking? fle in Dogs," Proceedings of the 38th Annual Veterinary Orthopedic Society Meeting, Snowmass, 5-12 March 2011.

[13] D. Hulse, W. Hyman and B. Beale, "Determination of Isometric Points for Placement of a Lateral Suture in Treatment of the Cranial Cruciate Ligament Deficient Stifle," Veterinary and Comparative Orthopedics and Traumatology, Vol. 23, No. 3, 2010, pp. 163-167.

[14] J. T. Hudson, M. R. Slater, L. Taylor, et al., “Assessing Repeatability and Validity of a Visual Analogue Scale Questionnaire for use in Assessing Pain and Lameness in Dogs," American Journal of Veterinary Research, Vol. 65, No. 12, 2004, pp. 1634-1643. doi:10.2460/ajvr.2004.65.1634

[15] D. Hulse, B. Saunders, B. Beale, et al., "Extra-Articular Stabilization of the Cranial Cruciate Deficient Stifle with Anchor Systems," Tieräztliche Praxis Kleintiere, Vol. 39, No. K, 2011, pp. 363-367.

[16] K. Thieman, J. L. Tomlinson, D. B. Fox, et al., "Effect of Meniscal Release on Rate of Subsequent Meniscal Tears and Owner-Assessed Outcome in Dogs with Cruciate Disease Treated with Tibial Plateau Leveling Osteotomy," Veterinary Surgery, Vol. 35, No. 8, 2006, pp. 705710. doi:10.1111/j.1532-950X.2006.00214.x

[17] M. B. Mueller, H. H. Fredrich, E. Steinhauser, et al., "Biomechanical Evaluation of Different Suture Anchors for the Stabilization of Anterior Labrum Lesions," Arthroscopy, Vol. 21, No. 5, 2005, pp. 611-619. doi:10.1016/j.arthro.2005.01.012

[18] D. C. Meyer, R. W. Nyffeler, S. F. Fucentese, et al., "Failure of Suture Material at Suture Anchor Eyelets," Arthroscopy, Vol. 18, No. 9, 2002, pp. 1013-1019. doi:10.1053/jars.2002.36115

[19] S. A. Aktay and M. P. Kowaleski, "Analysis of Suture Anchor Eyelet Position on Suture Failure Load," Veterinary Surgery, Vol. 40, No. 4, 2010, pp. 418-422. doi:10.1111/j.1532-950X.2011.00834.X

[20] F. A. Barbar, M. A. Herbert, R. C. Beavis, et al., "Suture Anchor Materials, Eyelets, and Designs: Update 2008," Arthroscopy, Vol. 24, No. 8, 2008, pp. 859-867. doi:10.1016/j.arthro.2008.03.006

7) Pain when suddenly turning while walking?

AFTER surgical repair and rehabilitation

1) What is quality of life now since surgery?

2) What is willingness to play voluntarily?

3) How is activity during the day?

4) Stiffness at the being of the day?

5) Stiffness at the end of the day?

6) Indication of lameness when walking on surgery limb?

7) Pain when suddenly turning while walking? 